
\title{
$\angle 3$ Research S Suare \\ Feasibility of Robotic Surgery in Comparison to the conventional Laparoscopic and Open Procedures in Urology: A Systematic Review
}

\author{
Afra Zahid \\ King Edward Medical College: King Edward Medical University \\ Muhammad Ayyan ( $\nabla$ iamayyan77@gmail.com ) \\ Minaam Farooq \\ King Edward Medical College: King Edward Medical University \\ Huzaifa Ahmad Cheema \\ King Edward Medical College: King Edward Medical University \\ Abia Shahid \\ King Edward Medical College: King Edward Medical University \\ Faiza Naeem \\ King Edward Medical College: King Edward Medical University \\ Muhammad Abdullah llyas \\ King Edward Medical College: King Edward Medical University \\ Shehreen Sohail \\ University of Central Punjab
}

King Edward Medical College: King Edward Medical University https://orcid.org/0000-0002-4023-7956

Research Article

Keywords: Robotic Surgical Procedures, Urology, Surgical Procedures, Prostatectomy

Posted Date: February 17th, 2022

DOI: https://doi.org/10.21203/rs.3.rs-1357100/v1

License: (c) (1) This work is licensed under a Creative Commons Attribution 4.0 International License. Read Full License 


\section{Abstract \\ PURPOSE}

To establish the feasibility of robotic surgical procedures in urology in terms of the applications, pros and cons as well as the postoperative and oncological outcomes while comparing it with the conventional approaches.

\section{METHODS}

A systematic search of electronic databases was performed to identify Randomized Controlled Trials and Cohort studies on Robot Assisted urological surgical procedures in comparison with the conventional methods. The quality assessment of included studies was performed using the Newcastle-Ottawa Scale and the revised Cochrane "Risk of Bias" tool. A qualitative narrative synthesis of the data extracted from the studies was performed and presented in tabulated form.

\section{RESULTS}

After screening, 34 studies were included in our review (3 Randomized Controlled Trials and 31 Cohort studies). Robot Assisted Prostatectomy appears to be associated with lower estimated blood loss and shorter length of hospital stay. For Robot Assisted Cystectomy, the results suggest longer operative time and fewer complications. Robot Assisted Radical Nephrectomy was found to be associated with fewer perioperative complications and longer mean operative time while Robot Assisted Partial Nephrectomy was associated with less positive surgical margins, no recurrence and reduced need of postoperative analgesia. The mean operative time was longer while length of stay was shorter for the robotic approach in inguinal lymphadenectomy and ureteral re-implantation.

\section{CONCLUSION}

The feasibility of Robot Assisted surgery varied for different outcome measures as well as for different procedures. Some common advantages were shorter length of stay, lesser blood loss and fewer complications while the drawbacks included longer operative time.

\section{Introduction}

The use of Artificial Intelligence (AI) in the field of surgery has been increasing over the past few decades. The first robot ever used for surgery was the Unimation PUMA 200 to assist a neurosurgical intervention performed by Kwoh et al. in 1985[1] while the first urological procedure for which a robot was used was transurethral prostate resection, performed by the assistance of PROBOT in 1989 [2, 3]. AESOP (Automated Endoscopic System for Optimal Positioning) was the first surgical robotic system that became FDA approved in 1993 and commercially available for use in surgical procedures, including those in the field of 
urology[4]. The ZEUS system was developed by Computer Motion Corporation (Goleta, CA) and introduced in the market in 1998. It was initially designed for use in procedures of cardiology but later on its use was extended to other fields too. However, it was discontinued in 2003[5]. The da Vinci surgical system was developed by Intuitive Surgical, Sunnyvale, CA, USA, and approved by the FDA for human use in 2000, and for prostate surgery in 2001[6].

Since then, the da Vinci system has been used in cardiothoracic, gastrointestinal, gynecologic, otolaryngologic and urologic procedures. In the field of urology, the first radical prostatectomy was performed in Germany and reported in literature by Abbou et al. in France[7] while the first nephrectomy was reported by Guillonneau et al[8]. The robot assisted laparoscopic procedures in the field of urology being performed nowadays include prostatectomy, cystectomy, nephrectomy, pyeloplasty, nephroureterectomy, ureteric reimplantation, inguinal lymphadenectomy and adrenalectomy.

The use of robots in surgical procedures has been increasing due to the advantages over the conventional surgical techniques including lower death rate, fewer complications, fewer transfusions, shorter length of stay, three-dimensional view of the operative field, and ergonomic benefits for the surgeon[9]

Previous systematic reviews on the subject target specific robot assisted procedures in urology including cystectomy[10, 11], nephrectomy[11, 12], nephroureterectomy[13] and prostatectomy[14]. Our systematic review aims at comprehensively compiling the information on all the commonly performed robot assisted procedures in the field of urology.

Our objective is to establish the feasibility of robot assisted procedures in terms of the applications, pros and cons, postoperative and oncological outcomes as well as cost effectiveness while comparing it with the more conventional open and laparoscopic approaches.

\section{Material And Methods}

This systematic review was conducted according to the methods recommended by the Cochrane Handbook for Systematic Reviews of Interventions[15] and written according to the PRISMA statement[16]. Ethical approval was not required for the study. This review was registered in The International Prospective Register of Systematic Reviews (PROSPERO) (Registration Number: CRD42021256623).

\section{ELIGIBILITY CRITERIA}

The studies included were in accordance to the following criteria: 1) The study designs included were Randomized Controlled Trials (RCTs) and Cohort Studies. 2) The studies published in English language during the years 2000 to 2021. 3) The articles including one or more of the following interventions regardless of the cause of the intervention: Robot Assisted Prostatectomy, Robot Assisted Cystectomy, Robot Assisted Radical Nephrectomy, Robot Assisted Partial Nephrectomy, Robot Assisted Laparoscopic Nephroureterectomy, Robot Assisted Laparoscopic Ureteral Reimplantation, Robot Assisted Adrenalectomy and Robot Assisted Inguinal Lymphadenectomy. 4) The studies that compared the robotic method to the conventional open and laparoscopic methods. 5) The studies conducted on human populations irrespective 
of the age. 6) The articles including patients belonging to any age group who underwent surgical interventions for urological conditions. 7) The articles on the feasibility of robotic surgery in the field of urology in terms of the outcome measures (primary, secondary and additional).

The studies were excluded according to the following criteria: 1) Systematic reviews, meta-analysis and other study designs except Randomized Controlled Trials (RCTs) and Cohort Studies. 2) Unpublished articles and those published in languages other than English. 3) Studies conducted on non-human populations. 4) The studies not dealing with robotic interventions. 5) The articles that did not deal with urological procedures. 6) The articles that did not deal with the feasibility of robotic surgery in terms of the outcome measures (primary, secondary and additional). 7) The studies that did not compare the robotic method to the conventional open and laparoscopic methods.

\section{INFORMATION SOURCES}

Studies were selected by searching electronic databases and scrutinizing the reference list of articles (citation tracking). Electronic searches of the following online resources were run:

- Electronic databases: Cochrane Central Register of Controlled Trials (CENTRAL, via The Cochrane Library), MEDLINE (via PubMed) and ScienceDirect.

- Grey literature sources: Open Access Theses and Dissertations (OATD), OpenGrey and Google Scholar.

The search was run on 5 August 2021. No publication restrictions were applied to the search. The studies published in English language between 2000 and present were sought. The database search was updated on 20 August 2021 just before the final analyses and further studies retrieved for inclusion.

\section{SEARCH STRATEGY}

The database search was done using the terms "Robotic surgical procedures", "Robotic surgery", "Urology" "Robot Assisted Prostatectomy", "Robot Assisted Cystectomy" etc. and their combinations.

The detailed search strategy is presented in Table 1. 
Table 1

Search strategy for MEDLINE (PubMed format)

\begin{tabular}{|c|c|}
\hline NUMBER & SEARCH TERMS \\
\hline$\# 1$ & Feasibility [All fields] \\
\hline \#2 & Advantages [All Fields] \\
\hline \#3 & Disadvantages [All Fields] \\
\hline \#4 & Cost* $^{\star}$ All Fields] \\
\hline \#5 & "Costs and Cost Analysis" [mh] \\
\hline \#6 & "Robotic Surgical Procedures/adverse effects" [mh] \\
\hline \#7 & "Robotic Surgical Procedures/mortality" [mh] \\
\hline \#8 & \#1 OR \#2 OR \#3 OR \#4 OR \#5 OR \#6 OR \#7 \\
\hline \#9 & Robotic Surgery [All fields] \\
\hline$\# 10$ & Robot Assisted Prostatectomy [All Fields] \\
\hline$\# 11$ & Robot Assisted Cystectomy [All Fields] \\
\hline$\# 12$ & Robot Assisted Radical Nephrectomy [All Fields] \\
\hline$\# 13$ & Robot Assisted Partial Nephrectomy [All Fields] \\
\hline$\# 14$ & Robot Assisted Laparoscopic Nephroureterectomy [All Fields] \\
\hline \#15 & Robot Assisted Laparoscopic Ureteral Reimplantation [All Fields] \\
\hline$\# 16$ & Robot Assisted Adrenalectomy [All Fields] \\
\hline \#17 & Robot Assisted Inguinal Lymphadenectomy [All Fields] \\
\hline$\# 18$ & \#9 OR \#10 OR \#11 OR \#12 OR \#13 OR \#14 OR \#15 OR \#16 OR \#17 \\
\hline \#19 & Urology [All Fields] \\
\hline \#20 & "Urology/surgery" [mh] \\
\hline \#21 & \#19 OR \#20 \\
\hline \#22 & \#8 AND \#18 AND \#21 \\
\hline
\end{tabular}

\section{STUDY SELECTION}

After the database search, the articles were imported to EndNote X9 Software and de-duplication was done. Two independent reviewers screened the articles, initially according to the title and abstract and later based on the full text. The articles were selected based on the inclusion criteria. Any conflict during the screening process was resolved by discussion. In case of unresolved disagreements, it was planned that the third author would make the decision. 


\section{DATA COLLECTION PROCESS AND DATA ITEMS}

Data extraction was performed by the two reviewers independently. The data was extracted into a structured Microsoft Excel sheet and it consisted of two categories: study variables and outcome variables. Study variables included Author ID, year of publishing, study design, type of intervention, years of intervention and number of patients. The outcome variables included primary, secondary and additional outcomes.

\section{PRIMARY OUTCOMES}

The primary outcomes were: (i) oncological outcomes (For example: positive surgical margins, recurrence rate and mean lymph node yield); (ii) functional outcomes (For example: time to flatus, renal function (reduction of GFR), urinary and sexual function); and (iii) complications.

\section{SECONDARY OUTCOMES}

The secondary outcome measures comprised of: (i) mortality; (ii) length of hospital stay; (iii) blood loss; (iv) mean operative time; (v) warm ischemia time; and (vi) need for postoperative analgesia.

The additional outcome was cost effectiveness.

\section{RISK OF BIAS (QUALITY) ASSESSMENT For Cohort Studies}

The Newcastle-Ottawa Scale (NOS) checklist[17] was used. It consists of three categories, namely Selection, Comparability and Representativeness, on the basis of which the cohort studies were divided into three levels of scoring: poor quality ( 1 to 4 stars), fair quality ( 5 to 7 stars) and good quality (8 to 9 stars).

\section{For Randomized Controlled Trials (RCTs)}

The quality assessment of the Randomized Controlled Trials was performed using the revised Cochrane "Risk of Bias" tool for randomized trials (RoB 2.0)[18]. RoB 2.0 consists of five specific domains: (1) bias arising from the randomization process; (2) bias due to deviations from the intended interventions; (3) bias due to missing outcome data; (4) bias in measurement of the outcome; and (5) bias in the selection of the reported result.

Using RoB 2.0, each specific outcome was assessed and its overall Risk of Bias was found (low; some concerns; high). The overall Risk of Bias was derived using the highest RoB levels in any of the domains that were tested.

The quality assessment of each study was performed by two review authors independently. Any disagreements were resolved by discussion. In case of unresolved disagreements, a third review author was consulted to make the decision.

DATA SYNTHESIS 
A qualitative synthesis of the data extracted from the included studies was performed. The data was presented in a tabulated form and included study variables, outcome variables, quality assessment and the conclusions of each individual study. Meta-analyses could not be conducted because of the heterogeneity of interventions, participants and outcome measures.

\section{Results}

\section{STUDY SELECTION}

A total of 12,088 records were found through databases searching. After de-duplication, 10,631 records were screened on the basis of title and abstract, from which we reviewed 151 full-text documents, and finally included 30 articles. Later, 25 studies were identified through citation searching, out of which 18 were assessed for eligibility, and 4 were finally included in the review. Hence, a total of 34 studies were included in our review. [Figure 1]

\section{STUDY CHARACTERISTICS}

34 studies, published between the years 2002 to 2020, were included in our systematic review, of which 31 (91.1\%) were cohort studies while 3 (8.9\%) were Randomized Controlled Trials (RCTs). Out of the included studies, 6 (17.6\%) dealt with Robot Assisted Radical Prostatectomy[19-24], 6 (17.6\%) dealt with Robot Assisted Cystectomy[25-30], 4 (11.7\%) dealt with Robot Assisted Radical Nephrectomy[31-34], 7 (20.5\%) dealt with Robot Assisted Partial Nephrectomy[35-41], 2 (5.8\%) dealt with Robot Assisted Laparoscopic Nephroureterectomy[42, 43] ,4 (11.7\%) dealt with Robot Assisted Laparoscopic Ureteral Reimplantation[4447], 3 (8.9\%) dealt with Robot Assisted Adrenalectomy[48-50] and 2 (5.8\%) dealt with Robot Assisted Inguinal Lymphadenectomy[51,52]. The study characteristics are summarized in Table 2. 
Table 2

Study Characteristics

\begin{tabular}{|c|c|c|c|c|c|}
\hline $\begin{array}{l}\text { Author ID } \\
\text { [Reference] }\end{array}$ & $\begin{array}{l}\text { Year of } \\
\text { Publishing }\end{array}$ & $\begin{array}{l}\text { Study } \\
\text { Design }\end{array}$ & Type of Intervention & $\begin{array}{l}\text { Years of } \\
\text { Intervention }\end{array}$ & Number of patients \\
\hline \multicolumn{6}{|c|}{ Robot-Assisted Prostatectomy } \\
\hline \multirow{2}{*}{$\begin{array}{l}\text { Mani } \\
\text { Menon et al. }\end{array}$} & \multirow[t]{2}{*}{2002} & \multirow{2}{*}{$\begin{array}{l}\text { Cohort } \\
\text { Study }\end{array}$} & \multirow{2}{*}{$\begin{array}{l}\text { Conventional } \\
\text { radical retropubic } \\
\text { prostatectomy } \\
\text { (RRP) and Robot- } \\
\text { assisted anatomic } \\
\text { prostatectomy } \\
\text { (RAP) }\end{array}$} & \multirow{2}{*}{$\begin{array}{l}\text { March } \\
2001 \text { to } \\
\text { August } \\
2001\end{array}$} & $\mathrm{RRP}=30$ \\
\hline & & & & & $\mathrm{RAP}=30$ \\
\hline \multirow{2}{*}{$\begin{array}{l}\text { Christian } \\
\text { Bolenz et al. }\end{array}$} & \multirow[t]{2}{*}{2009} & \multirow{2}{*}{$\begin{array}{l}\text { Cohort } \\
\text { Study }\end{array}$} & \multirow[b]{2}{*}{$\begin{array}{l}\text { Robotic-Assisted } \\
\text { Laparoscopic } \\
\text { Radical } \\
\text { Prostatectomy } \\
\text { (RALP), } \\
\text { Laparoscopic } \\
\text { Radical } \\
\text { Prostatectomy } \\
\text { (LRP), and open } \\
\text { Retropubic Radical } \\
\text { Prostatectomy } \\
\text { (RRP). }\end{array}$} & \multirow{2}{*}{$\begin{array}{l}2003 \text { to } \\
2008\end{array}$} & RALP $=262$ \\
\hline & & & & & $\begin{array}{l}\text { LRP }=220 \mathrm{RRP}= \\
161\end{array}$ \\
\hline \multirow{2}{*}{$\begin{array}{l}\text { Jim C. Hu et } \\
\text { al. }\end{array}$} & \multirow[t]{2}{*}{2009} & \multirow{2}{*}{$\begin{array}{l}\text { Cohort } \\
\text { Study }\end{array}$} & \multirow{2}{*}{$\begin{array}{l}\text { Minimally Invasive } \\
\text { Radical } \\
\text { Prostatectomy } \\
\text { (MIRP) and open } \\
\text { Retropubic Radical } \\
\text { Prostatectomy } \\
\text { (RRP) }\end{array}$} & \multirow{2}{*}{$\begin{array}{l}2003 \text { to } \\
2007\end{array}$} & MIRP $=1938$ \\
\hline & & & & & $\mathrm{RRP}=6899$ \\
\hline \multirow{2}{*}{$\begin{array}{l}\text { Jong Wook } \\
\text { Park et al. }\end{array}$} & \multirow[t]{2}{*}{2011} & \multirow{2}{*}{$\begin{array}{l}\text { Cohort } \\
\text { Study }\end{array}$} & \multirow[b]{2}{*}{$\begin{array}{l}\text { Laparoscopic } \\
\text { Radical } \\
\text { Prostatectomy } \\
\text { (LRP) and Robot- } \\
\text { Assisted } \\
\text { Laparoscopic } \\
\text { Prostatectomy } \\
\text { (RALP) }\end{array}$} & \multirow{2}{*}{$\begin{array}{l}2007 \text { to } \\
2009\end{array}$} & $\mathrm{LRP}=62$ \\
\hline & & & & & $\mathrm{RALP}=44$ \\
\hline $\begin{array}{l}\text { Guillaume } \\
\text { Ploussard } \\
\text { et al. }\end{array}$ & 2012 & $\begin{array}{l}\text { Cohort } \\
\text { Study }\end{array}$ & $\begin{array}{l}\text { Robot-Assisted } \\
\text { Radical } \\
\text { Prostatectomy } \\
\text { (RALP) and } \\
\text { Laparoscopic } \\
\text { Radical } \\
\text { Prostatectomy } \\
\text { (LRP) }\end{array}$ & $\begin{array}{l}2001 \text { to } \\
2011\end{array}$ & $\begin{array}{l}\mathrm{LRP}=1377 \text { RALP }= \\
1009\end{array}$ \\
\hline
\end{tabular}




\begin{tabular}{|c|c|c|c|c|c|}
\hline $\begin{array}{l}\text { Author ID } \\
\text { [Reference] }\end{array}$ & $\begin{array}{l}\text { Year of } \\
\text { Publishing }\end{array}$ & $\begin{array}{l}\text { Study } \\
\text { Design }\end{array}$ & Type of Intervention & $\begin{array}{l}\text { Years of } \\
\text { Intervention }\end{array}$ & Number of patients \\
\hline $\begin{array}{l}\text { Fransesco } \\
\text { Porpiglia et } \\
\text { al. }\end{array}$ & 2016 & $\begin{array}{l}\text { Randomized } \\
\text { Controlled } \\
\text { Trial }\end{array}$ & $\begin{array}{l}\text { Robot-assisted } \\
\text { radical } \\
\text { prostatectomy } \\
\text { (RARP) and } \\
\text { Laparoscopic } \\
\text { radical } \\
\text { prostatectomy } \\
\text { (LRP) }\end{array}$ & $\begin{array}{l}2010 \text { to } \\
2011\end{array}$ & 120 \\
\hline \multicolumn{6}{|c|}{ Robot-Assisted Cystectomy } \\
\hline \multirow{2}{*}{$\begin{array}{l}\text { Casey K Ng } \\
\text { et al. }\end{array}$} & \multirow[t]{2}{*}{2010} & \multirow{2}{*}{$\begin{array}{l}\text { Cohort } \\
\text { Study }\end{array}$} & \multirow{2}{*}{$\begin{array}{l}\text { Open Radical } \\
\text { Cystectomy (ORC) } \\
\text { and Robot-Assisted } \\
\text { Radical Cystectomy } \\
\text { (RARC) }\end{array}$} & \multirow{2}{*}{$\begin{array}{l}2002 \text { to } \\
2008\end{array}$} & $\mathrm{ORC}=104$ \\
\hline & & & & & $\mathrm{RARC}=83$ \\
\hline \multirow{2}{*}{$\begin{array}{l}\text { Bernard H. } \\
\text { Bochner et } \\
\text { al. }\end{array}$} & \multirow[t]{2}{*}{2015} & \multirow{2}{*}{$\begin{array}{l}\text { Randomized } \\
\text { Controlled } \\
\text { Trial }\end{array}$} & \multirow{2}{*}{$\begin{array}{l}\text { Open Radical } \\
\text { Cystectomy (ORC) } \\
\text { and Robot-Assisted } \\
\text { Radical Cystectomy } \\
\text { (RARC) }\end{array}$} & \multirow{2}{*}{$\begin{array}{l}2010 \text { to } \\
2013\end{array}$} & $\mathrm{ORC}=58$ \\
\hline & & & & & $\mathrm{RARC}=60$ \\
\hline \multirow{3}{*}{$\begin{array}{l}\text { Muhammad } \\
\text { Shamim } \\
\text { Khan et al. }\end{array}$} & \multirow[t]{3}{*}{2016} & \multirow{3}{*}{$\begin{array}{l}\text { Randomized } \\
\text { Controlled } \\
\text { Trial }\end{array}$} & \multirow{3}{*}{$\begin{array}{l}\text { Open Radical } \\
\text { Cystectomy (ORC), } \\
\text { Laparoscopic } \\
\text { Radical Cystectomy } \\
\text { (LRC), and Robot- } \\
\text { Assisted Radical } \\
\text { Cystectomy (RARC). }\end{array}$} & \multirow{3}{*}{$\begin{array}{l}2009 \text { to } \\
2012\end{array}$} & $\mathrm{ORC}=20$ \\
\hline & & & & & $\mathrm{RARC}=20$ \\
\hline & & & & & $L R C=20$ \\
\hline \multirow{2}{*}{$\begin{array}{l}\text { Agata } \\
\text { Gastecka et } \\
\text { al. }\end{array}$} & \multirow[t]{2}{*}{2018} & \multirow{2}{*}{$\begin{array}{l}\text { Cohort } \\
\text { Study }\end{array}$} & \multirow{2}{*}{$\begin{array}{l}\text { Laparoscopic } \\
\text { Radical Cystectomy } \\
\text { (LRC) and Robot- } \\
\text { Assisted Radical } \\
\text { Cystectomy (RARC) }\end{array}$} & \multirow{2}{*}{$\begin{array}{l}2016 \text { to } \\
2018\end{array}$} & $\mathrm{LRC}=37$ \\
\hline & & & & & $\mathrm{RARC}=52$ \\
\hline \multirow{2}{*}{$\begin{array}{l}\text { Shiwei } \\
\text { Zhang et al. }\end{array}$} & \multirow[t]{2}{*}{2019} & \multirow{2}{*}{$\begin{array}{l}\text { Cohort } \\
\text { Study }\end{array}$} & \multirow{2}{*}{$\begin{array}{l}\text { Laparoscopic } \\
\text { Radical Cystectomy } \\
\text { (LRC) and Robot- } \\
\text { Assisted Radical } \\
\text { Cystectomy (RARC) }\end{array}$} & \multirow{2}{*}{$\begin{array}{l}2010 \text { to } \\
2019\end{array}$} & $\mathrm{LRC}=126$ \\
\hline & & & & & $\mathrm{RARC}=172$ \\
\hline \multirow{2}{*}{$\begin{array}{l}\text { Janet } \\
\text { Baack } \\
\text { Kukreja et } \\
\text { al. }\end{array}$} & \multirow[t]{2}{*}{2020} & \multirow{2}{*}{$\begin{array}{l}\text { Cohort } \\
\text { Study }\end{array}$} & \multirow{2}{*}{$\begin{array}{l}\text { Open Radical } \\
\text { Cystectomy (ORC) } \\
\text { and Robot-Assisted } \\
\text { Radical Cystectomy } \\
\text { (RARC) }\end{array}$} & \multirow{2}{*}{$\begin{array}{l}2007 \text { to } \\
2015\end{array}$} & $\mathrm{RARC}=100$ \\
\hline & & & & & $\mathrm{ORC}=96$ \\
\hline
\end{tabular}




\begin{tabular}{|c|c|c|c|c|c|}
\hline $\begin{array}{l}\text { Author ID } \\
\text { [Reference] }\end{array}$ & $\begin{array}{l}\text { Year of } \\
\text { Publishing }\end{array}$ & $\begin{array}{l}\text { Study } \\
\text { Design }\end{array}$ & Type of Intervention & $\begin{array}{l}\text { Years of } \\
\text { Intervention }\end{array}$ & Number of patients \\
\hline \multirow{3}{*}{$\begin{array}{l}\text { Tanya } \\
\text { Nazemi et } \\
\text { al. }\end{array}$} & \multirow[t]{3}{*}{2006} & \multirow{3}{*}{$\begin{array}{l}\text { Cohort } \\
\text { Study }\end{array}$} & \multirow{3}{*}{$\begin{array}{l}\text { Robotic Radical } \\
\text { Nephrectomy (RRN), } \\
\text { Laparoscopic } \\
\text { Radical } \\
\text { Nephrectomy (LRN), } \\
\text { and Open Radical } \\
\text { Nephrectomy (ORN) }\end{array}$} & \multirow{3}{*}{$\begin{array}{l}2000 \text { to } \\
2004\end{array}$} & ORN $=18$ \\
\hline & & & & & $\mathrm{RRN}=6$ \\
\hline & & & & & $L R N=33$ \\
\hline \multirow{2}{*}{$\begin{array}{l}\text { Ashok K. } \\
\text { Hemal et al. }\end{array}$} & \multirow[t]{2}{*}{2008} & \multirow{2}{*}{$\begin{array}{l}\text { Cohort } \\
\text { Study }\end{array}$} & \multirow{2}{*}{$\begin{array}{l}\text { Robotic Radical } \\
\text { Nephrectomy (RRN) } \\
\text { and Laparoscopic } \\
\text { Radical } \\
\text { Nephrectomy (LRN). }\end{array}$} & \multirow{2}{*}{$\begin{array}{l}2006 \text { to } \\
2007\end{array}$} & $\mathrm{RRN}=15$ \\
\hline & & & & & $\mathrm{LRN}=15$ \\
\hline \multirow{2}{*}{$\begin{array}{l}\text { In Gab } \\
\text { Jeong et al. }\end{array}$} & \multirow[t]{2}{*}{2017} & \multirow{2}{*}{$\begin{array}{l}\text { Cohort } \\
\text { Study }\end{array}$} & \multirow{2}{*}{$\begin{array}{l}\text { Robotic Radical } \\
\text { Nephrectomy (RRN) } \\
\text { and Laparoscopic } \\
\text { Radical } \\
\text { Nephrectomy (LRN) }\end{array}$} & \multirow{2}{*}{$\begin{array}{l}2003 \text { to } \\
2015\end{array}$} & $\mathrm{RRN}=5180$ \\
\hline & & & & & LRN $=18573$ \\
\hline \multirow{2}{*}{$\begin{array}{l}\text { Boris } \\
\text { Gershman } \\
\text { et al. }\end{array}$} & \multirow[t]{2}{*}{2018} & \multirow{2}{*}{$\begin{array}{l}\text { Cohort } \\
\text { Study }\end{array}$} & \multirow{2}{*}{$\begin{array}{l}\text { Robot-Assisted } \\
\text { Laparoscopic } \\
\text { Radical } \\
\text { Nephrectomy } \\
\text { (RALRN) and } \\
\text { Laparoscopic } \\
\text { Radical } \\
\text { Nephrectomy (LRN) }\end{array}$} & \multirow{2}{*}{$\begin{array}{l}2010 \text { to } \\
2013\end{array}$} & RALRN = 4926 \\
\hline & & & & & LRN $=3390$ \\
\hline \multicolumn{6}{|c|}{ Robot-Assisted Partial Nephrectomy } \\
\hline \multirow{2}{*}{$\begin{array}{l}\text { Leslie A } \\
\text { Deane et al. }\end{array}$} & \multirow[t]{2}{*}{2008} & \multirow{2}{*}{$\begin{array}{l}\text { Cohort } \\
\text { Study }\end{array}$} & \multirow{2}{*}{$\begin{array}{l}\text { Robot-assisted } \\
\text { Laparoscopic } \\
\text { Partial } \\
\text { Nephrectomy } \\
\text { (RLPN) and } \\
\text { Laparoscopic } \\
\text { Partial } \\
\text { Nephrectomy (LPN) }\end{array}$} & \multirow{2}{*}{$\begin{array}{l}2003 \text { to } \\
2007\end{array}$} & $\mathrm{LPN}=12$ \\
\hline & & & & & RLPN $=10$ \\
\hline \multirow{2}{*}{$\begin{array}{l}\text { Ali Riza } \\
\text { Kural et al. }\end{array}$} & \multirow[t]{2}{*}{2009} & \multirow[t]{2}{*}{$\begin{array}{l}\text { Cohort } \\
\text { Study }\end{array}$} & \multirow{2}{*}{$\begin{array}{l}\text { Laparoscopic } \\
\text { Partial } \\
\text { Nephrectomy (LPN) } \\
\text { and Robot-Assisted } \\
\text { Partial } \\
\text { Nephrectomy } \\
\text { (RAPN) }\end{array}$} & \multirow{2}{*}{$\begin{array}{l}2003 \text { to } \\
2009\end{array}$} & $\mathrm{LPN}=20$ \\
\hline & & & & & RAPN $=11$ \\
\hline \multirow{2}{*}{$\begin{array}{l}\text { Brian M } \\
\text { Benway et } \\
\text { al. }\end{array}$} & \multirow[t]{2}{*}{2009} & \multirow{2}{*}{$\begin{array}{l}\text { Cohort } \\
\text { Study }\end{array}$} & \multirow{2}{*}{$\begin{array}{l}\text { Laparoscopic } \\
\text { Partial } \\
\text { Nephrectomy (LPN) } \\
\text { and Robot-Assisted } \\
\text { Partial } \\
\text { Nephrectomy } \\
\text { (RAPN) }\end{array}$} & \multirow{2}{*}{$\begin{array}{l}2004 \text { to } \\
2008\end{array}$} & LPN-118 \\
\hline & & & & & RAPN $=129$ \\
\hline
\end{tabular}




\begin{tabular}{|c|c|c|c|c|c|}
\hline $\begin{array}{l}\text { Author ID } \\
\text { [Reference] }\end{array}$ & $\begin{array}{l}\text { Year of } \\
\text { Publishing }\end{array}$ & $\begin{array}{l}\text { Study } \\
\text { Design }\end{array}$ & Type of Intervention & $\begin{array}{l}\text { Years of } \\
\text { Intervention }\end{array}$ & Number of patients \\
\hline \multirow{2}{*}{$\begin{array}{l}\text { Agnes J } \\
\text { Wang et al. }\end{array}$} & \multirow[t]{2}{*}{2009} & \multirow{2}{*}{$\begin{array}{l}\text { Cohort } \\
\text { Study }\end{array}$} & Laparoscopic & \multirow{2}{*}{$\begin{array}{l}\text { Not } \\
\text { mentioned }\end{array}$} & LPN $=62$ \\
\hline & & & $\begin{array}{l}\text { Partial } \\
\text { Nephrectomy (LPN) } \\
\text { and Robotic- } \\
\text { assisted Partial } \\
\text { Nephrectomy (RPN) }\end{array}$ & & $\mathrm{RPN}=40$ \\
\hline \multirow{2}{*}{$\begin{array}{l}\text { Sangchul } \\
\text { Lee et al. }\end{array}$} & \multirow[t]{2}{*}{2011} & \multirow{2}{*}{$\begin{array}{l}\text { Cohort } \\
\text { Study }\end{array}$} & Robot-assisted & \multirow{2}{*}{$\begin{array}{l}2003 \text { to } \\
2010\end{array}$} & $\mathrm{OPN}=234$ \\
\hline & & & $\begin{array}{l}\text { Nephrectomy (RPN) } \\
\text { and Open Partial } \\
\text { Nephrectomy (OPN) }\end{array}$ & & $\mathrm{RPN}=69$ \\
\hline \multirow{2}{*}{$\begin{array}{l}\text { Phillip M. } \\
\text { Pierorazio } \\
\text { et al. }\end{array}$} & \multirow[t]{2}{*}{2011} & \multirow{2}{*}{$\begin{array}{l}\text { Cohort } \\
\text { Study }\end{array}$} & Robot-Assisted & \multirow{2}{*}{$\begin{array}{l}2006 \text { to } \\
2011\end{array}$} & LPN $=102$ \\
\hline & & & $\begin{array}{l}\text { LaparoscopIC } \\
\text { Partial } \\
\text { Nephrectomy } \\
\text { (RALPN) and } \\
\text { Laparoscopic } \\
\text { Partial } \\
\text { Nephrectomy (LPN) }\end{array}$ & & RALPN $=48$ \\
\hline \multirow{2}{*}{$\begin{array}{l}\text { Ali Khalifeh } \\
\text { et al. }\end{array}$} & \multirow[t]{2}{*}{2012} & \multirow{2}{*}{$\begin{array}{l}\text { Cohort } \\
\text { Study }\end{array}$} & Laparoscopic & \multirow{2}{*}{$\begin{array}{l}2002 \text { to } \\
2012\end{array}$} & $\mathrm{RPN}=261$ \\
\hline & & & $\begin{array}{l}\text { Nephrectomy (LPN) } \\
\text { and Robotic- } \\
\text { assisted Partial } \\
\text { Nephrectomy (RPN) }\end{array}$ & & LPN $=231$ \\
\hline \multicolumn{6}{|c|}{ Robot-Assisted Laparoscopic Nephroureterectomy } \\
\hline \multirow{2}{*}{$\begin{array}{l}\text { Vincent } \\
\text { Trudeau et } \\
\text { al. }\end{array}$} & \multirow[t]{2}{*}{2014} & \multirow{2}{*}{$\begin{array}{l}\text { Cohort } \\
\text { Study }\end{array}$} & Robotic-Assisted & \multirow{2}{*}{$\begin{array}{l}2008 \text { to } \\
2010\end{array}$} & LNU $=1199$ \\
\hline & & & $\begin{array}{l}\text { Nephroureterectomy } \\
\text { (RANU) and } \\
\text { Laparoscopic } \\
\text { radical } \\
\text { Nephroureterectomy } \\
\text { (LNU) }\end{array}$ & & RANU $=715$ \\
\hline \multirow{3}{*}{$\begin{array}{l}\text { Hakmin Lee } \\
\text { et al. }\end{array}$} & \multirow[t]{3}{*}{2019} & \multirow{3}{*}{$\begin{array}{l}\text { Cohort } \\
\text { Study }\end{array}$} & Open & \multirow{3}{*}{$\begin{array}{l}2004 \text { to } \\
2017\end{array}$} & $\mathrm{ONU}=161$ \\
\hline & & & $\begin{array}{l}\text { Nephroureterectomy } \\
\text { (ONU), }\end{array}$ & & $\mathrm{LNU}=137$ \\
\hline & & & $\begin{array}{l}\text { LaparoscopIC } \\
\text { Nephroureterectomy } \\
\text { (LNU), and Robotic- } \\
\text { Assisted } \\
\text { Laparoscopic } \\
\text { Nephroureterectomy } \\
\text { (RALNU) }\end{array}$ & & RALNU $=124$ \\
\hline
\end{tabular}




\begin{tabular}{|c|c|c|c|c|c|}
\hline $\begin{array}{l}\text { Author ID } \\
\text { [Reference] }\end{array}$ & $\begin{array}{l}\text { Year of } \\
\text { Publishing }\end{array}$ & $\begin{array}{l}\text { Study } \\
\text { Design }\end{array}$ & Type of Intervention & $\begin{array}{l}\text { Years of } \\
\text { Intervention }\end{array}$ & Number of patients \\
\hline \multirow{2}{*}{$\begin{array}{l}\text { Ryan P. } \\
\text { Smith et al. }\end{array}$} & \multirow[t]{2}{*}{2011} & \multirow{2}{*}{$\begin{array}{l}\text { Cohort } \\
\text { Study }\end{array}$} & \multirow{2}{*}{$\begin{array}{l}\text { Robotic Extravesical } \\
\text { Ureteral } \\
\text { Reimplantation } \\
\text { (RALUR) and Open } \\
\text { Ureteral } \\
\text { Reimplantation }\end{array}$} & \multirow{2}{*}{$\begin{array}{l}2006 \text { to } \\
2009\end{array}$} & RALUR $=25$ \\
\hline & & & & & $\begin{array}{l}\text { Open ureteral } \\
\text { reimplantation = } 25\end{array}$ \\
\hline \multirow{2}{*}{$\begin{array}{l}\text { John L. } \\
\text { Schomburg } \\
\text { et al. }\end{array}$} & \multirow[t]{2}{*}{2014} & \multirow{2}{*}{$\begin{array}{l}\text { Cohort } \\
\text { Study }\end{array}$} & \multirow{2}{*}{$\begin{array}{l}\text { Robot-assisted } \\
\text { laparoscopic } \\
\text { Extravesical Ureteral } \\
\text { Reimplantation } \\
\text { (RALUR) and Open } \\
\text { Extravesical Ureteral } \\
\text { Reimplantation }\end{array}$} & \multirow{2}{*}{$\begin{array}{l}2008 \text { to } \\
2010\end{array}$} & RALUR $=20$ \\
\hline & & & & & $\begin{array}{l}\text { Open ureteral } \\
\text { reimplantation }=20\end{array}$ \\
\hline \multirow[t]{2}{*}{$\begin{array}{l}\text { Michael P. } \\
\text { Kurtz et ai. }\end{array}$} & \multirow[t]{2}{*}{2016} & \multirow[t]{2}{*}{$\begin{array}{l}\text { Cohort } \\
\text { Study }\end{array}$} & \multirow{2}{*}{$\begin{array}{l}\text { Robot-assisted } \\
\text { laparoscopic } \\
\text { Extravesical Ureteral } \\
\text { Reimplantation } \\
\text { (RALUR) and Open } \\
\text { Extravesical Ureteral } \\
\text { Reimplantation }\end{array}$} & $\begin{array}{l}2003 \text { to } \\
2013\end{array}$ & $\begin{array}{l}\text { Open ureteral } \\
\text { reimplantation = } \\
1494\end{array}$ \\
\hline & & & & & RALUR = 108 \\
\hline \multirow{2}{*}{$\begin{array}{l}\text { Rodolfo A. } \\
\text { Elizondo et } \\
\text { al. }\end{array}$} & \multirow[t]{2}{*}{2020} & \multirow[t]{2}{*}{$\begin{array}{l}\text { Cohort } \\
\text { Study }\end{array}$} & \multirow{2}{*}{$\begin{array}{l}\text { Robot-assisted } \\
\text { laparoscopic } \\
\text { Extravesical Ureteral } \\
\text { Reimplantation } \\
\text { (RALUR) and Open } \\
\text { Extravesical Ureteral } \\
\text { Reimplantation }\end{array}$} & $\begin{array}{l}2013 \text { to } \\
2015\end{array}$ & $\begin{array}{l}\text { Open ureteral } \\
\text { reimplantation = } 38\end{array}$ \\
\hline & & & & & RALUR $=38$ \\
\hline \multicolumn{6}{|c|}{ Robot-Assisted Adrenalectomy } \\
\hline \multirow{2}{*}{$\begin{array}{l}\text { Luis Felipe } \\
\text { Brandao et } \\
\text { al. }\end{array}$} & \multirow[t]{2}{*}{2014} & \multirow{2}{*}{$\begin{array}{l}\text { Cohort } \\
\text { Study }\end{array}$} & \multirow{2}{*}{$\begin{array}{l}\text { Robotic } \\
\text { Adrenalectomy (RA) } \\
\text { and Laparoscopic } \\
\text { Adrenalectomy (LA) }\end{array}$} & \multirow{2}{*}{$\begin{array}{l}2004 \text { to } \\
2013\end{array}$} & $\mathrm{RA}=30$ \\
\hline & & & & & $\mathrm{LA}=46$ \\
\hline \multirow{2}{*}{$\begin{array}{l}\text { Arman } \\
\text { Arghami et } \\
\text { al. }\end{array}$} & \multirow[t]{2}{*}{2015} & \multirow[t]{2}{*}{$\begin{array}{l}\text { Cohort } \\
\text { Study }\end{array}$} & \multirow{2}{*}{$\begin{array}{l}\text { Single Port Robotic } \\
\text { Adrenalectomy } \\
\text { (SPRA) and } \\
\text { Laparoscopic } \\
\text { Adrenalectomy (LA) }\end{array}$} & \multirow[t]{2}{*}{$\begin{array}{l}2006 \text { to } \\
2013\end{array}$} & SPRA $=16$ \\
\hline & & & & & $\mathrm{LA}=16$ \\
\hline \multirow{2}{*}{$\begin{array}{l}\text { Kai } \\
\text { Alexander } \\
\text { Probst et al. }\end{array}$} & \multirow[t]{2}{*}{2016} & \multirow{2}{*}{$\begin{array}{l}\text { Cohort } \\
\text { Study }\end{array}$} & \multirow{2}{*}{$\begin{array}{l}\text { Robot-Assisted } \\
\text { Laparoscopic } \\
\text { Adrenalectomy } \\
\text { (RALA) and Open } \\
\text { Adrenalectomy (OA) }\end{array}$} & \multirow{2}{*}{$\begin{array}{l}2001 \text { to } \\
2005\end{array}$} & RALA $=56$ \\
\hline & & & & & $O A=33$ \\
\hline
\end{tabular}




\begin{tabular}{|c|c|c|c|c|c|}
\hline $\begin{array}{l}\text { Author ID } \\
\text { [Reference] }\end{array}$ & $\begin{array}{l}\text { Year of } \\
\text { Publishing }\end{array}$ & $\begin{array}{l}\text { Study } \\
\text { Design }\end{array}$ & Type of Intervention & $\begin{array}{l}\text { Years of } \\
\text { Intervention }\end{array}$ & Number of patients \\
\hline $\begin{array}{l}\text { Amitabh } \\
\text { Singh et al. }\end{array}$ & 2018 & $\begin{array}{l}\text { Cohort } \\
\text { Study }\end{array}$ & $\begin{array}{l}\text { Robot-Assisted } \\
\text { Inguinal } \\
\text { Lymphadenectomy } \\
\text { (RAIL) and Open } \\
\text { Inguinal } \\
\text { Lymphadenectomy }\end{array}$ & $\begin{array}{l}2012 \text { to } \\
2016\end{array}$ & $\begin{array}{l}\text { RAIL = } 51 \\
\text { Open Inguinal } \\
\text { Lymphadenectomy } \\
=100\end{array}$ \\
\hline $\begin{array}{l}\text { Hualiang Yu } \\
\text { et al. }\end{array}$ & 2019 & $\begin{array}{l}\text { Cohort } \\
\text { Study }\end{array}$ & $\begin{array}{l}\text { Robot-Assisted } \\
\text { Inguinal } \\
\text { Lymphadenectomy } \\
\text { (RAIL) and Open } \\
\text { Inguinal } \\
\text { Lymphadenectomy }\end{array}$ & $\begin{array}{l}2013 \text { to } \\
2017\end{array}$ & $\begin{array}{l}\text { RAIL = } 9 \\
\text { Open Inguinal } \\
\text { Lymphadenectomy } \\
=10\end{array}$ \\
\hline
\end{tabular}

\section{RISK OF BIAS}

Newcastle-Ottawa Scale (NOS) was used in order to perform the quality assessment of the 31 cohort studies included in the review. $29(93.5 \%)^{19-23,25,28-30,32-40,42-52}$ of the included cohort studies were assessed to have a good quality (low risk of bias) while $2(6.5 \%)[31,41]$ were assessed to have a fair quality (medium risk of bias). The most common methodological flaws were inadequate representativeness of the exposed cohort, non-adjustment for the confounders and inadequate duration of the follow-up. The findings are summarized in Table 3.

The quality assessment of the 3 Randomized Controlled Trials (RCTs) was performed using the revised Cochrane "Risk of Bias" tool for randomized trials (RoB 2.0). The overall risk of bias was assessed to be "low" for 1 (33.3\%) study[27] and "some concerns" for the other $2(66.6 \%)[24,26]$. The most common methodological flaws were lack of blinding of participants and outcome assessors, improper allocation concealment, deviations from the intended interventions and lack of information regarding pre-specified analysis plan. The RoB plot was created using the Robvis tool[53]. [Figure 2]

\section{RESULTS OF INDIVIDUAL STUDIES}

The results of each individual study included in the review are presented in Table 4. 


\section{Author ID, Summary of Findings \\ Year}

\section{Robot-Assisted Prostatectomy}

Mani $\quad$ Robot-assisted prostatectomy was found to take a longer time (2.3 hours for

Menon et al, conventional radical retropubic prostatectomy versus 4.8 hours for robot-assisted

2002 prostatectomy $(p<0.001))$ but associated with less blood loss $(970 \mathrm{~mL}$ for RRP versus $329 \mathrm{~mL}$ for RAP $(p<0.001))$, early discharge from the hospital and less postoperative pain (mean pain score on postoperative day 1 was 7 in the RRP group and 4 in the RAP group $(p=0.05)$. The complications and oncological outcomes were found to be similar to those of radical retropubic prostatectomy.

Christian The robot-assisted surgery was found to be more expensive than open and laparoscopic Bolenz et al, approaches (RALP: \$6752, LRP: \$5687, RRP: \$4437, p < 0.001).

2009

Jim C. Hu et al, 2009

Robot-assisted surgery was found to be associated with less need of transfusions $(2.7 \%$ versus $20.8 \%$; $p<0.001)$, less risk of respiratory $(4.3 \%$ versus $6.6 \% ; p=0.004)$, and other complications $(4.3 \%$ versus $5.6 \% ; p=0.03)$ but higher risk of genitourinary complications $(4.7 \%$ versus $2.1 \% ; p=0.001)$ like incontinence and erectile dysfunction as compared to the open approach.

Jong Wook

Park et al,

2011

Both robot-assisted and laparoscopic prostatectomy had similar safety and efficacy but the robotic approach was associated with faster recovery of continence, although the continence rate at 12 months became similar (95\% for LRP versus $94.4 \%$ for RALP, $p=$ 1.00).

Guillaume Ploussard et al, 2012

The robot-assisted approach was found to be associated with shorter operative time (129 versus 175 min; $p<0.001$ ), less blood loss (515 versus $800 \mathrm{ml} ; p<0.001$ ), and shorter hospital stays (4.0 versus 5.7 days; $p<0.001$ ) as compared to the pure laparoscopic approach. Continence was unaffected by the type of procedure performed while there was an early recovery of potency by the robot-assisted approach (After 6 months, 20\% patients were potent after LRP and 42\% after RALP. After 12 months, 31.6\% of patients were potent after LRP and $57.7 \%$ after RALP)

Fransesco Porpiglia et al, 2016

Robot-assisted prostatectomy was associated with better functional outcomes in terms of recovery of continence $(p<0.021)$ and potency $(p<0.028)$ but was found to be similar to the laparoscopic approach in terms of the oncological outcomes and major complications.

\section{Robot-Assisted Cystectomy}

Casey K. Ng Robot-assisted surgery was found to be associated with a lesser risk of complications et al, 2010 ( $59 \%$ for the open group versus $41 \%$ for the robot-assisted group; $p=0.04$ ), both major and minor as compared to the open approach but the types of complications were similar in both groups.

Bernard $\mathrm{H}$. Bochner et al, 2015
Robot-assisted radical cystectomy (RARC) was found to be associated with no significant advantage over standard open radical cystectomy with respect to 90-d complications, length of stay, 3-month and 6-month quality of life, or costs. 


\section{Author ID, Summary of Findings \\ Year}

Muhammad There was no significant difference in the 30-day or 90-day complications between the Shamim different approaches. However, the robot-assisted approach was found to be associated Khan et al, with greater mean operating time $(p<0.001)$, positive surgical margins as well as the 2016 greatest risk of recurrence as compared to the open and laparoscopic approaches (ORC: 2 of 19 ,

RARC: 5 of 19, LRC: 3 of $18 ; p=0.5$ )

Agata

Gastecka et al, 2018

Robot-assisted cystectomy was found to be less cost-effective as compared to the laparoscopic approach (LRC: €3336; RARC: €4052; $p<0.001)$. The higher cost was attributed to the robotic instruments (LRC: €130; RARC: €1166; per case)

Shiwei

Zhang et al,

The robotic approach was associated with some perioperative benefits as compared to 2019

the laparoscopic approach. RARC group had lesser operative time $(p<0.001)$, lesser blood loss $(p<0.001)$, lower intraoperative transfusion rate $(p<0.05)$, shorter hospital stays ( $p<0.001)$, and a lower 90-day complication rate compared with the LRC group, but there was no difference in the rate of readmissions between the two approaches.

Janet Baack Kukreja et al, 2020

The robot-assisted approach was more expensive as compared to the open approach but was associated with lesser costs per quality-adjusted life years (RARC was \$17 000 more expensive, but associated with an increase of 0.32 QALYs)

\section{Robot-Assisted Radical Nephrectomy}

Tanya The robot-assisted approach was said to be associated with lower blood loss $(p=0.01)$

Nazemi et al, 2006 and analgesia use (open nephrectomy compared to robotic and hand-assisted laparoscopic methods at $75 \%$ versus $0 \%$ and $14 \%$, for the robotic and hand-assisted laparoscopic methods respectively $(p=0.0035)$ ) and shorter hospital stay (3 vs. 5 days for the open method $(p<0.01))$ but greater operative time $(345(246-548)$ minutes compared to the open method, $202(116-382)$ minutes $(p=0.02))$. The oncological outcomes were similar in all the approaches.

Ashok K. The robot-assisted approach was similar to the laparoscopic approach in terms of the Hemal et al, oncological and perioperative complications but was associated with a slightly longer 2008 operative time (221 versus 175.3 minutes in robot-assisted and laparoscopic methods, respectively, $p=0.001)$.

In Gab

Jeong et al,

2017

The robot-assisted approach was associated with higher costs as compared to the other approaches for nephrectomy (\$19,530 versus \$16,851; difference, $\$ 2678 ; 95 \%$ Cl, $\$ 838$ to $\$ 4519)$

Boris

Gershman

et al, 2018

Robot assisted nephrectomy was found to be associated with lower intraoperative (0.9\% versus $1.8 \% ; p<0.001)$ and postoperative $(20.4 \%$ versus $27.2 \% ; p<0.001)$ complications but higher costs (median $\$ 16,207$ versus $\$ 15,037 ; p<0.001$ )

\section{Robot Assisted Partial Nephrectomy}

Leslie A

Deane et al, 2008
Robot-assisted partial nephrectomy was not associated with any significant difference from the laparoscopic approach in terms of blood loss $(115 \mathrm{~mL}$ versus $198 \mathrm{~mL} ; \mathrm{p}=$ $0.169)$, total operative time (228.7 minutes versus 289.5 minutes; $p=0.102)$, and warm ischemia time (32.1 minutes versus 35.3 minutes; $p=0.501)$. There was no reported case of recurrence or any major complications. 


\section{Author ID, Summary of Findings
Year}

Ali Riza

Kural et al, 2009

Robot-assisted partial nephrectomy was associated with the advantages of threedimensional vision and easier suturing over the traditional laparoscopic approach. The mean warm ischemia time was found to be significantly shorter in the RAPN group (27.3 minutes for the RAPN group and 35.8 for the LPN group) $(p=0.02)$. Some demerits included higher costs and the need for surgeons with experience in the field.

Brian M Benway et al, 2009
Robot-assisted partial nephrectomy provided comparable pathological outcomes and morbidity as compared to traditional laparoscopy. However, it was associated with reduced blood loss ( 155 for RAPN versus $196 \mathrm{ml}$ for $L P N, p=0.03$ ), warm ischemia time (19.7 versus 28.4 minutes, $p<0.0001)$, and length of hospital stay ( 2.4 versus 2.7 days, $p<0.0001)$. It was also found to be supposedly superior to the laparoscopic approach while dealing with more complex tumors.
Agnes J Wang et al, 2009
The robot-assisted procedure was comparable to the laparoscopic partial nephrectomy in terms of postoperative outcomes but was associated with shortened warm ischemia time (19 versus 25 minutes, $p=0.03$ ). Some disadvantages included higher cost, use of extra trocars (4.6 versus $3.2, p=0.01$ ), and the more invasive nature of the robotic approach.
Sangchul Lee et al, 2011
Both the robotic and open approaches were associated with similar changes in GFR (83.3 for RPN versus $79.6 \mathrm{~mL} / \mathrm{min} / 1.73 \mathrm{~m}^{2}$ for OPN, $\mathrm{p}=0.146$ ) but the robotic surgery was preferred due to shorter length of hospital stay $(6.2$ versus 8.9 days, $p<0.001)$ and lesser analgesic use $(0.3$ versus 0.9 ampules, $p<0.001)$ while the open approach was favorable in terms of duration of operation (192 versus 143 minutes, $p<0.001)$ and ischemia time $(22.99$ versus 18.14 minutes, $p<0.001)$.
Phillip M. Pierorazio et al, 2011
Robot-assisted partial nephrectomy was found to be associated with the advantages of having shorter operative (152 versus 193 minutes, $p<0.001)$ and ischemia times (14.0 versus 18.0 minutes, $p<0.001$ ) as well as reduced blood loss (122 versus $245 \mathrm{~mL}, p=$ 0.001 ) as compared to the traditional laparoscopic approach.
Ali Khalifeh et al, 2012
The robot-assisted approach was found to have the advantages of having lower intraoperative complications (2.6\% for the robotic approach versus $5.6 \%$ for the laparoscopic approach, each $\mathrm{p}<0.001)$, lower postoperative complications $(24.53 \%$ for the robotic approach versus $32.03 \%$ for the laparoscopic approach, $p=0.004$ ) and lower risk of positive surgical margins (2.9\% for the robotic procedure versus $5.6 \%$ for the laparoscopic procedure, $p<0.001$ ) as compared to the conventional laparoscopic method of surgery.

\section{Robot-Assisted Laparoscopic Nephroureterectomy}

Vincent Trudeau et al, 2014

Hakmin Lee et al, 2019
The robot-assisted approach was found to be more costly $(\$ 23,235$ for the robotic approach versus $\$ 17,637$ for the laparoscopic approach; $p<0.001)$ but associated with lesser complications (11.9\% in the robotic approach versus $18.2 \%$ in the laparoscopic approach; $p<0.001$ ) as compared to the traditional laparoscopic approach.

The laparoscopic and robotic showed some better perioperative outcomes (all p-values $>0.05$ ) after radical nephroureterectomy as compared to the open approach in patients with non-metastatic UTUC. The recurrence rate was comparable in all the groups $(p=$ 0.279).

\section{Robot Assisted Laparoscopic Ureteral Reimplantation}




\section{Author ID, Summary of Findings
Year}

Ryan P.

Smith et al,

2011

Mean operative time was longer by $12 \%$ in the robotic group as compared to the controls $(p<0.05)$. The mean length of stay (33 versus 53 hours) and the use of postoperative analgesia were significantly less in the robotic group $(p<0.001)$. Robot-assisted surgery for ureteral reimplantation had similar success rates as compared to the open approach ( $97 \%$ for robot-assisted laparoscopy versus $100 \%$ for open reimplantation).

John L.

Schomburg

et al, 2014

The clinical outcomes for the robotic approach were almost the same as those for the

Michael P. open approach while the postoperative analgesic requirement was significantly reduced for the robotic approach (RALUR: $0.14 \mathrm{mg} / \mathrm{kg}$, open: $0.25 \mathrm{mg} / \mathrm{kg}, \mathrm{p}=0.021$ ).

Kurtz et al,

2016

RALUR was associated with a significantly higher rate of complications $(13.0 \%$ of RALUR versus $4.5 \%$ of OUR; $p=0.0037)$ as well as higher direct costs $(\$ 9,128$ for RALUR versus $\$ 7,273$ for OUR; $p=0.0043$ ) as compared to the open approach.

Rodolfo A. Elizondo et The clinical outcomes (open $94.8 \%$ versus robotic $94.8 \%$ ) for both the open and robotic al, 2020 approaches were found to be comparable.

The overall total charges including the costs of hospitalization were similar between the OUR $(\$ 21,461)$ and RALUR groups with $(\$ 22,860)$ and without cystoscopy $(\$ 21,437)(p=$ 0.34 and $p=0.53$ respectively)

\section{Robot Assisted Adrenalectomy}

Luis Felipe

Brandao et al, 2014
A significantly lower median estimated blood loss was recorded for the robotic group (50 $\mathrm{ml}$ [IQR: 50] in the robotic group versus $100 \mathrm{ml}$ [IQR: 288] in the laparoscopic group; $\mathrm{p}=$ 0.02 ). The distribution of pheochromocytomas in the Laparoscopic group was significantly higher than in the Robotic group (43.5\% in Laparoscopic Adrenalectomy versus $16.7 \%$ in Robotic Adrenalectomy; $p=0.02$ )
Arman Arghami et al, 2015
Robot-assisted adrenalectomy was found to be a feasible approach since it was associated with lesser use of analgesics (43 mg in Robotic Adrenalectomy versus $84 \mathrm{mg}$ in Laparoscopic Adrenalectomy group, $p<0.001)$ but comparable costs $(84 \% \pm 14 \%$ in Robotic Adrenalectomy versus $100 \% \pm 16 \%$ in Laparoscopic Adrenalectomy)

Kai The robot-assisted approach was found to be feasible for the dissection of adrenal Alexander tumors. Although it was associated with longer operative time (128.5 $\pm 46.5 \mathrm{~min}$ for Probst et al, RALA versus $102.2 \pm 44.5 \mathrm{~min}$ for $\mathrm{OA}$ ), the overall hospital stay was found to be shorter 2016 for the robotic approach $(6.8 \pm 1.2$ days for RALA versus $11.1 \pm 4.8$ days for OA).

\section{Robot-Assisted Inguinal Lymphadenectomy}

Amitabh The robot-assisted approach was found to be associated with lower morbidity and Singh et al, comparable lymph node yield (13 in Robot-assisted procedure versus 12.5 in open 2018 inguinal lymph node dissection) as compared to the open approach.

Hualiang Yu The robot-assisted approach was found to be associated with fewer intraoperative and et al, 2019 postoperative complications while achieving the desired outcomes.

\section{RESULTS OF SYNTHESES}

A qualitative narrative synthesis of the data extracted from the included studies was performed and presented in the form of tables. There was not a scope for a meta-analysis, given the likely range of 
interventions, participants and outcomes. The results for the feasibility of each surgical intervention according to the outcomes have been presented in Table 5 . 
Table 5

Findings of studies according to outcome measures

Robot-Assisted Prostatectomy

$\begin{array}{ll}\text { OUTCOME } & \text { NO. OF } \\ & \text { PARTICIPANTS CONCLUSIONS } \\ & \text { (STUDIES) }\end{array}$

Positive 266 (Three

Surgical Studies)

No significant difference was found between the robotic and other

Margins

$\begin{array}{ll}\text { Recovery of } & 9063 \text { (Three } \\ \text { continence } & \text { Studies) }\end{array}$

approaches

Two studies reported faster recovery of continence in the robotic approach as compared to the other approaches while one reported greater urinary incontinence for the robotic procedure.

$\begin{array}{lll}\begin{array}{l}\text { Recovery of } \\ \text { sexual }\end{array} & \begin{array}{l}9063 \text { (Three } \\ \text { function }\end{array} & \begin{array}{l}\text { Two studies reported faster recovery of potency in the robotic } \\ \text { approach while one reported greater erectile dysfunction for the } \\ \text { robotic approach. }\end{array}\end{array}$

Complications 11389 (Four Studies)

Two studies reported lesser complications for the robotic approach while one reported no difference. According to one study, the rate of genitourinary complications was higher in the robotic approach.

Postoperative 8837 (One analgesia Study)

Reduced need for postoperative analgesia as compared to the other approaches.

$\begin{array}{ll}\text { Estimated } & 11283(\text { Three } \quad \text { Less as compared to other approaches } \\ \text { blood loss } & \text { Studies) }\end{array}$

$\begin{array}{lll}\begin{array}{l}\text { Mean } \\ \text { operative time }\end{array} & \begin{array}{l}2552 \text { (Three } \\ \text { Studies) }\end{array} & \begin{array}{l}\text { Two studies reported greater mean operative t } \\ \text { reported shorter operative time. }\end{array} \\ \text { Length of stay } & \begin{array}{l}11223 \text { (Two } \\ \text { Studies) }\end{array} & \text { Shorter as compared to the other approaches. }\end{array}$

\section{Robot-Assisted Cystectomy}

$\begin{array}{ll}\text { OUTCOME } & \text { NO. OF } \\ & \text { PARTICIPANTS } \\ & \text { (STUDIES) }\end{array}$

$\begin{array}{lll}\text { Positive } & 247 \text { (Two } & \text { One study reported a higher rate of positive surgical margins while } \\ \text { Surgical } & \text { Studies) } & \text { one reported comparable rates to the other approaches. }\end{array}$

Margins

$\begin{array}{ll}\text { Mean Lymph } & 247 \text { (Two } \\ \text { node Yield } & \text { Studies) }\end{array}$

According to one study, the lymph node yield was comparable in the robotic and other approaches while one study reported greater lymph node yield as compared to the laparoscopic approach but lesser as compared to the open approach.

Recurrence $\quad 60$ (One Study) Greater as compared to the other approaches.

Rate

Mortality $\quad 60$ (One Study)

According to one study, the mortality for the robotic-assisted procedure was found to be higher than the open approach but lower than the laparoscopic approach. 


\section{Robot-Assisted Prostatectomy}

$\begin{array}{lll}\text { Complications } & \begin{array}{l}247 \text { (Two } \\ \text { Studies) }\end{array} & \begin{array}{l}\text { Fewer as compared to the open approach while one study reported } \\ \text { thromboembolic complications. }\end{array} \\ \begin{array}{l}\text { Estimated } \\ \text { Blood Loss }\end{array} & \begin{array}{l}187 \text { (One } \\ \text { Study) }\end{array} & \text { Less as compared to the other methods. }\end{array}$

$\begin{array}{ll}\text { Mean } & 247 \text { (Two } \quad \text { Greater as compared to the other approaches. } \\ \text { Operative } & \text { Studies) }\end{array}$

Time

$\begin{array}{ll}\text { Length of } & 187 \text { (One Shorter median length of stay. } \\ \text { Stay } & \text { Study) }\end{array}$

\section{Robot-Assisted Radical Nephrectomy}

$\begin{array}{ll}\text { OUTCOME } & \text { NO. OF } \\ & \text { PARTICIPANTS CONCLUSIONS } \\ & \text { (STUDIES) }\end{array}$

\begin{tabular}{|c|c|c|}
\hline $\begin{array}{l}\text { Oncological } \\
\text { Outcomes }\end{array}$ & $\begin{array}{l}102 \text { (One } \\
\text { Study) }\end{array}$ & Comparable to the other approaches. \\
\hline Complications & $\begin{array}{l}8316 \text { (One } \\
\text { Study) }\end{array}$ & Fewer perioperative complications for the robotic approach. \\
\hline $\begin{array}{l}\text { Postoperative } \\
\text { analgesia }\end{array}$ & $\begin{array}{l}132 \text { (Two } \\
\text { Studies) }\end{array}$ & $\begin{array}{l}\text { According to one study, the need for postoperative analgesia was } \\
\text { less as compared to the conventional methods while one study } \\
\text { reported comparable postoperative analgesia use. }\end{array}$ \\
\hline $\begin{array}{l}\text { Estimated } \\
\text { Blood Loss }\end{array}$ & $\begin{array}{l}132 \text { (Two } \\
\text { Studies) }\end{array}$ & $\begin{array}{l}\text { According to one study, the estimated blood loss was less as } \\
\text { compared to the conventional methods while one study reported } \\
\text { comparable blood loss. }\end{array}$ \\
\hline $\begin{array}{l}\text { Mean } \\
\text { Operative } \\
\text { Time }\end{array}$ & $\begin{array}{l}132 \text { (Two } \\
\text { Studies) }\end{array}$ & Greater as compared to the conventional methods. \\
\hline $\begin{array}{l}\text { Length of } \\
\text { Stay }\end{array}$ & $\begin{array}{l}132 \text { (Two } \\
\text { Studies) }\end{array}$ & $\begin{array}{l}\text { According to one study, the length of stay was shorter as compared } \\
\text { to the conventional methods while one study reported a comparable } \\
\text { length of stay. }\end{array}$ \\
\hline
\end{tabular}

\section{Robot-Assisted Partial Nephrectomy}

$\begin{array}{ll}\text { OUTCOME } & \text { NO. OF } \\ & \text { PARTICIPANTS CONCLUSIONS } \\ & \text { (STUDIES) }\end{array}$

\begin{tabular}{lll}
$\begin{array}{l}\text { Positive } \\
\text { Surgical } \\
\text { Margins }\end{array}$ & $\begin{array}{l}1175 \text { (Five } \\
\text { Studies) }\end{array}$ & $\begin{array}{l}\text { No or less positive surgical margins as compared to the other } \\
\text { approaches. }\end{array}$ \\
\hline $\begin{array}{l}\text { Recurrence } \\
\text { Rate }\end{array}$ & $\begin{array}{l}53 \text { (Two } \\
\text { Studies) }\end{array}$ & No recurrence after the robotic surgery. \\
\hline $\begin{array}{l}\text { Renal } \\
\text { Function } \\
\text { (GFR) }\end{array}$ & $\begin{array}{l}463 \text { (Two } \\
\text { Studies) }\end{array}$ & $\begin{array}{l}\text { According to one study, the decrease in GFR was less as compared } \\
\text { to the conventional methods while one study reported comparable } \\
\text { changes in GFR. }\end{array}$ \\
\hline
\end{tabular}




\section{Robot-Assisted Prostatectomy}

$\begin{array}{lll}\text { Complications } & 998 \text { (Five } & \begin{array}{l}\text { Two studies reported decreased complications as compared to the } \\ \text { other methods while two reported one complication each for the } \\ \text { robotic approach. According to one study, the robotic approach was } \\ \text { associated with increased complications, the most common being } \\ \text { urinary leakage while one reported comparable intraoperative } \\ \text { complications. }\end{array}\end{array}$

Postoperative 303 (One $\quad$ Reduced need for postoperative analgesia.

Analgesia Study)

$\begin{array}{lll}\text { Estimated } & 855 \text { (Six } & \begin{array}{l}\text { Three studies reported reduced blood loss for the robotic approach } \\ \text { while according to three studies, the blood loss was similar for all } \\ \text { approaches. }\end{array}\end{array}$

$\begin{array}{lll}\begin{array}{l}\text { Mean } \\ \text { Operative }\end{array} & \begin{array}{l}1347 \text { (Seven } \\ \text { Studies) }\end{array} & \begin{array}{l}\text { According to four studies, the operative time was shorter for the } \\ \text { robotic approach while the others reported comparable, or even } \\ \text { increased operative time. }\end{array}\end{array}$

$\begin{array}{lll}\begin{array}{l}\text { Length of } \\ \text { Stay }\end{array} & \begin{array}{l}380 \text { (Three } \\ \text { Studies) }\end{array} & \begin{array}{l}\text { The length of stay was found to be shorter for the robotic method } \\ \text { by two studies but one reported similar results for the different } \\ \text { approaches. }\end{array} \\ \begin{array}{l}\text { Warm } \\ \text { Ischemia } \\ \text { Time }\end{array} & \begin{array}{l}1347 \text { (Seven } \\ \text { Studies) }\end{array} & \begin{array}{l}\text { According to five studies, the warm ischemia time was shorter for } \\ \text { the robotic approach while the others reported comparable, or even } \\ \text { increased warm ischemia time. }\end{array}\end{array}$

\section{Robot-Assisted Laparoscopic Nephroureterectomy}

$\begin{array}{ll}\text { OUTCOME } & \text { NO. OF } \\ & \text { PARTICIPANTS CONCLUSIONS } \\ & \text { (STUDIES) }\end{array}$

$\begin{array}{ll}\text { Mortality } & 1914 \text { (One Less for the robotic approach. } \\ \text { Study) }\end{array}$

$\begin{array}{lll}\text { Complications } & \begin{array}{l}1957 \text { (Two } \\ \text { Studies) }\end{array} \quad \text { Fewer for the robotic approach as compared to the other methods. }\end{array}$

Estimated
Blood Loss $\quad 43$ (One Study) $\begin{aligned} & \text { No significant difference in the blood loss for the different } \\ & \text { approaches. }\end{aligned}$

$\begin{array}{lll}\begin{array}{l}\text { Length of } \\ \text { Stay }\end{array} & \begin{array}{l}1957 \text { (Two } \\ \text { Studies) }\end{array} & \begin{array}{l}\text { According to one study, the length of stay was less as compared to } \\ \text { the conventional methods while one study reported comparable } \\ \text { outcomes. }\end{array}\end{array}$

\section{Robot Assisted Laparoscopic Ureteral Reimplantation}

$\begin{array}{ll}\text { OUTCOME } & \text { NO. OF } \\ & \text { PARTICIPANTS CONCLUSION } \\ & \text { (STUDIES) }\end{array}$

\begin{tabular}{lll} 
Complications & $\begin{array}{l}204 \text { (Two } \\
\text { Studies) }\end{array}$ & $\begin{array}{l}\text { Less for the robotic approach while one study reported increased } \\
\text { risk of vesicoureteral reflux. }\end{array}$ \\
\hline $\begin{array}{l}\text { Postoperative } \\
\text { analgesia }\end{array}$ & $\begin{array}{l}101 \text { (Two } \\
\text { Studies) }\end{array}$ & Reduced need for postoperative analgesia for the robotic approach. \\
\hline
\end{tabular}




\section{Robot-Assisted Prostatectomy}

\begin{tabular}{|c|c|c|}
\hline $\begin{array}{l}\text { Mean } \\
\text { Operative } \\
\text { Time }\end{array}$ & $\begin{array}{l}101 \text { (Two } \\
\text { Studies) }\end{array}$ & Increased for the robotic approach. \\
\hline $\begin{array}{l}\text { Length of } \\
\text { Stay }\end{array}$ & $\begin{array}{l}193 \text { (Two } \\
\text { Studies) }\end{array}$ & Shorter as compared to the conventional methods. \\
\hline
\end{tabular}

\section{Robot-Assisted Adrenalectomy}

$\begin{array}{ll}\text { OUTCOME } & \text { NO. OF } \\ & \text { PARTICIPANTS CONCLUSION } \\ & \text { (STUDIES) }\end{array}$

$\begin{array}{lll}\text { Complications } & 165 \text { (Two } & \text { Fewer complications in the robot-assisted method. } \\ \text { Studies) }\end{array}$

$\begin{array}{lll}\begin{array}{l}\text { Postoperative } \\ \text { analgesia }\end{array} & \begin{array}{l}108 \text { (Two } \\ \text { Studies) }\end{array} & \begin{array}{l}\text { Reduced or comparable need of postoperative analgesia as } \\ \text { compared to the other methods. }\end{array}\end{array}$

Estimated
Blood Loss (One Study) Less for the robotic approach.

$\begin{array}{lll}\begin{array}{l}\text { Mean } \\ \text { Operative }\end{array} & 194 \text { (Three } & \begin{array}{l}\text { Different studies reported variable results of the mean operative } \\ \text { time for the robotic approach as compared to the other methods. }\end{array}\end{array}$

Time

$\begin{array}{lll}\begin{array}{l}\text { Length of } \\ \text { Stay }\end{array} & \begin{array}{l}\text { Reduced length of stay for the robotic approach while one study } \\ \text { Studies) }\end{array} & \begin{array}{l}\text { Reduced la similar length of stay. } \\ \text { reported a }\end{array}\end{array}$

Robot-Assisted Inguinal Lymphadenectomy

$\begin{array}{ll}\text { OUTCOME } & \text { NO. OF } \\ & \text { PARTICIPANTS CONCLUSION } \\ & \text { (STUDIES) }\end{array}$

Complications 167 (Three Fewer complications as compared to the conventional methods. Studies)

$\begin{array}{ll}\text { Estimated } & 151 \text { (One } \quad \text { Similar for all the approaches. } \\ \text { Blood Loss } & \text { Study) }\end{array}$

$\begin{array}{ll}\text { Mean } & 164 \text { (Two } \quad \text { Longer for the robotic approach. } \\ \text { Operative } & \text { Studies) }\end{array}$

Time

Length of 154 (Two Shorter as compared to the conventional methods.

Stay Studies)

\section{Discussion}

The use of robots for surgical procedures has been gaining popularity, especially in certain fields including urology. This has become an important topic for researchers, and many studies have reported their superiority as compared to the conventional open and laparoscopic methods. Our study explains the 
differences between the robotic and other approaches in terms of outcome measures comprehensively, covering all the urological procedures.

The results of our systematic review suggest that Robot Assisted Prostatectomy is associated with reduced need of postoperative analgesia, lower estimated blood loss and shorter length of hospital stay as compared to the conventional methods. No significant difference in positive surgical margins (PSM) was found between the robotic and other approaches. Tang et al.[54] and Cao et al.[55] reported similar outcomes regarding estimated blood loss and length of hospital stay but Tang et al.[54] reported lower PSM rates for Robotic approach while Cao et al.[55] reported no significant difference in the PSM rates for the different approaches.

For Robot Assisted Cystectomy, the findings of our study suggest lesser blood loss, shorter median length of hospital stay, greater recurrence rate and longer operative time for the robotic procedure as compared to the conventional approaches. Our results were in accordance with other meta-analyses[56, 57] while Iwata et al. [58] reported no significant difference in the recurrence rate between robotic and other approaches.

Robot Assisted Radical Nephrectomy was found to be associated with fewer perioperative complications and longer mean operative time. This was in contrast to the findings of two meta-analyses[59,60], which reported comparable complications rates for the robotic and other approaches. The results of our review suggested no or less positive surgical margins, no recurrence and reduced need of postoperative analgesia for Robot Assisted Partial Nephrectomy as compared to the other approaches. The results of two previous meta-analyses $[61,62]$ suggested that the robotic method had no significant difference from the other methods regarding positive surgical margins (PSM) and recurrence rates.

The mean operative time was longer while length of stay was found to be shorter for the robotic approach in the procedures of inguinal lymphadenectomy and ureteral reimplantation as compared to their respective conventional approaches. According to the results of a study conducted in 2020[63], the robot assisted ureteral reimplantation was found to have longer operative time as well as longer time to discharge. The overall complications were less for the robotic approach in the procedures of inguinal lymphadenectomy, adrenalectomy and nephroureterectomy according to our study. Veccia et al.[64] reported similar outcome of lower complications for robot assisted nephroureterectomy while Tang et al.[65] reported comparable complications for adrenalectomy.

Our results are consistent with the fact that robot assisted surgeries, being minimally invasive, have the advantage of being associated with fewer complications, lesser blood loss, lesser need of postoperative analgesia and shorter length of hospital stay. The mean operative time was found to be longer for robot assisted surgeries due to extra time required for robot specific tasks, such as set-up of equipment and longer trocar entry time[66]. For some of the outcome measures, our results differed from those of other systematic reviews and meta-analyses because of the differences in the study settings and the surgeon skill set of the studies included. The differences can also be attributed to a bias in the selection of the studies that were included in the reviews. 
Most of the studies included in our review were limited by short periods of follow up and lack of randomization. Other limitations included small sample size and the retrospective nature of some studies, which introduced an inherent selection bias. Some of the included cohort studies were not controlled for confounders.

Our review was registered in the International Prospective Register of Systematic Reviews (PROSPERO). A highly sensitive search strategy was applied for the selection of articles and all the robotic surgical procedures in the field of urology have been included. There were certain limitations of the review process. A meta-analysis could not be conducted owing to the significant heterogeneity between the study results. Only the articles that were published in English language were included. The number of RCTs included was less, and so was the number of articles of each surgical procedure. The assessment and reporting of outcomes was not well standardized. However, we are confident that these limitations would not have an effect on the conclusions of the review.

According to our review, the robotic approach can be a good alternative to the conventional procedures due to the advantage of being associated with shorter length of hospital stay, lesser estimated blood loss, reduced need of postoperative analgesia and fewer perioperative and postoperative complications.

Our review can be used by patients, urologists and policy makers to make decisions regarding robotic surgical procedures by weighing the pros and cons and establishing its feasibility. There is an existing research gap when it comes to robotic surgery, especially in procedures like Inguinal Lymphadenectomy, Ureteral Reimplantation, Nephroureterectomy and Adrenalectomy.

More Randomized Controlled Trials (RCTs), especially those dealing with outcome measures like oncological and postoperative measures, and those with longer follow up duration are required to better ascertain the feasibility of robotic surgical procedures.

\section{Conclusion}

The feasibility of Robot Assisted surgery varied for different outcome measures as well as for different procedures. Some of the common advantages included shorter length of hospital stay, lesser estimated blood loss, reduced need of postoperative analgesia and fewer perioperative and postoperative complications while the disadvantages included longer operative time. The feasibility of Robot Assisted procedures can be better determined by more and larger clinical trials.

\section{Declarations}

\section{FUNDING}

We declare that no funds or grants were received during the preparation of this review.

\section{COMPETING INTERESTS}

We do not have any relevant financial or non-financial interests. 


\section{AUTHOR CONTRIBUTIONS}

Afra Zahid, Muhammad Ayyan, Huzaifa Ahmad Cheema and Minaam Farooq contributed to the conception and design of the review and screening of the articles to be included. Abia Shahid, Faiza Naeem, Abdullah Ilyas and Shehreen Sohail performed the analysis and interpretation of the included studies. All the authors contributed to the drafting, reviewing, and final approval of the manuscript.

\section{ETHICS APPROVAL}

No ethical approval was required for this study.

\section{Abbreviations And Acronyms}

Al- Artificial Intelligence

PUMA- Programmable Universal Manipulation Arm

AESOP-Automated Endoscopic System for Optimal Positioning

FDA- Food and Drug Administration

PRISMA- Preferred Reporting Items for Systematic Reviews and Meta-Analyses

PROSPERO- The International Prospective Register of Systematic Reviews

RCT- Randomized Controlled Trial

CENTRAL- Cochrane Central Register of Controlled Trials

MEDLINE-

OATD- Open Access Theses and Dissertations

GFR- Glomerular Filtration Rate

NOS- Newcastle-Ottawa Scale

RoB 2.0- Cochrane "Risk of Bias" tool for randomized trials

RRP- Radical Retropubic Prostatectomy

RAP- Robot-Assisted anatomic Prostatectomy

RALP- Robotic-Assisted Laparoscopic radical Prostatectomy

LRP- Laparoscopic Radical Prostatectomy

RRP- Retropubic Radical Prostatectomy 
MIRP- Minimally Invasive Radical Prostatectomy

ORC- Open Radical Cystectomy

RARC- Robot Assisted Radical Cystectomy

LRC- Laparoscopic Radical Cystectomy

RRN- Robotic Radical Nephrectomy

LRN- Laparoscopic Radical Nephrectomy

ORN- Open Radical Nephrectomy

RALRN- Robot-Assisted Laparoscopic Radical Nephrectomy

RLPN- Robot-assisted Laparoscopic Partial Nephrectomy

LPN- Laparoscopic Partial Nephrectomy

RPN- Robotic-assisted Partial Nephrectomy

OPN- Open Partial Nephrectomy

RALPN- Robot-Assisted Laparoscopic Partial Nephrectomy

RANU- Robotic-Assisted Nephroureterectomy

LNU- Laparoscopic radical Nephroureterectomy

ONU- Open Nephroureterectomy

RALNU- Robotic-Assisted Laparoscopic Nephroureterectomy

RALUR- Robotic Extravesical Ureteral Reimplantation

RA- Robotic Adrenalectomy

LA- Laparoscopic Adrenalectomy

SPRA- Single Port Robotic Adrenalectomy

RALA- Robot-Assisted Laparoscopic Adrenalectomy

OA- Open Adrenalectomy

RAlL- Robot-Assisted Inguinal Lymphadenectomy

QALY- Quality-Adjusted Life Year 
UTUC- Upper Tract Urothelial Cancer

IQR- Inpatient Quality Reporting

PSM- Positive Surgical Margins

\section{References}

1. Kwoh YS, Hou J, Jonckheere EA, Hayati S (1988) A robot with improved absolute positioning accuracy for CT guided stereotactic brain surgery. IEEE Trans Biomed Eng 35:153-160. https://doi.org/10.1109/10.1354

2. Davies BL, Hibberd RD, Coptcoat MJ, Wickham JEA (1989) A surgeon robot prostatectomy-a laboratory evaluation. J Med Eng Technol 13:273-277. https://doi.org/10.3109/03091908909016201

3. Davies BL, Hibberd RD, Ng WS et al (1991) The Development of a Surgeon Robot for Prostatectomies. Proc Inst Mech Eng Part H J Eng Med 205:35-38. https://doi.org/10.1243/PIME_PROC_1991_205_259_02

4. Unger SW, Unger HM, Bass RT (1994) AESOP robotic arm. Surg Endosc 8:1131

5. Thaly R, Shah K, Patel VR (2007) Applications of robots in urology. J Robot Surg 1:3-17. https://doi.org/10.1007/s11701-006-0003-9

6. Bullock K (1997) Medical renaissance. Trustee 50:5-6

7. Abbou CC, Hoznek A, Salomon L et al (2001) Laparoscopic radical prostatectomy with a remote controlled robot. J Urol 165. https://doi.org/10.1097/00005392-200106000-00027

8. Guillonneau B, Jayet C, Tewari A, Vallancien G (2001) Robot assisted laparoscopic nephrectomy. J Urol 166:200-201. https://doi.org/10.1016/S0022-5347(05)66111-3

9. Yu HY, Hevelone ND, Lipsitz SR et al (2012) Use, costs and comparative effectiveness of robotic assisted, laparoscopic and open urological surgery. J Urol 187:1392-1399.

https://doi.org/10.1016/j.juro.2011.11.089

10. Satkunasivam R, Tallman CT, Taylor JM et al (2019) Robot-assisted Radical Cystectomy Versus Open Radical Cystectomy: A Meta-analysis of Oncologic, Perioperative, and Complication-related outcomes. Eur Urol Oncol 2:443-447. https://doi.org/10.1016/j.euo.2018.10.008

11. Shekelle P, Maggard-gibbons M (2019) Robotic-assisted surgery in partial nephrectomy and cystectomy. Prospero

12. Crocerossa F, Carbonara U, Cantiello F et al (2021) Robot-assisted Radical Nephrectomy: A Systematic Review and Meta-analysis of Comparative Studies. Eur Urol 80:428-439. https://doi.org/10.1016/J.EURURO.2020.10.034

13. Veccia A, Antonelli A, Francavilla S et al (2019) Robotic versus other nephroureterectomy techniques: a systematic review and meta-analysis of over 87,000 cases. World J Urol 2019384 38:845-852. https://doi.org/10.1007/S00345-019-03020-1

14. Lai A, Dobbs RW, Talamini S et al (2020) Single port robotic radical prostatectomy: a systematic review. Transl Androl Urol 9:89805-89905. https://doi.org/10.21037/TAU.2019.11.05 
15. Cochrane Handbook for Systematic Reviews of Interventions | Cochrane Training. https://training.cochrane.org/handbook. Accessed 18 Nov 2021

16. Moher D, Liberati A, Tetzlaff J, Altman DG (2010) Preferred reporting items for systematic reviews and meta-analyses: the PRISMA statement. Int J Surg 8:336-341.

https://doi.org/10.1016/J.IJSU.2010.02.007

17. Bouhassira EE (2015) Ottawa Hospital Research Institute. In: The SAGE Encyclopedia of Stem Cell Research

18. Sterne JAC, Savović J, Page MJ et al (2019) RoB 2: A revised tool for assessing risk of bias in randomised trials. BMJ 366. https://doi.org/10.1136/bmj.|4898

19. Menon M, Tewari A, Baize B et al (2002) Prospective comparison of radical retropubic prostatectomy and robot-assisted anatomic prostatectomy: The Vattikuti Urology Institute experience. Urology 60:864-868. https://doi.org/10.1016/S0090-4295(02)01881-2

20. Bolenz C, Gupta A, Hotze T et al (2010) Cost Comparison of Robotic, Laparoscopic, and Open Radical Prostatectomy for Prostate Cancer. Eur Urol 57:453-458. https://doi.org/10.1016/j.eururo.2009.11.008

21. Hu JC, Gu X, Lipsitz SR et al (2009) Comparative effectiveness of minimally invasive vs open radical prostatectomy. JAMA - J Am Med Assoc 302:1557-1564. https://doi.org/10.1001/jama.2009.1451

22. Park JW, Won Lee H, Kim W et al (2011) Comparative assessment of a single surgeon's series of laparoscopic radical prostatectomy: Conventional versus robot-assisted. J Endourol 25:597-602. https://doi.org/10.1089/end.2010.0229

23. Ploussard G, De La Taille A, Moulin M et al (2014) Comparisons of the perioperative, functional, and oncologic outcomes after robot-assisted versus pure extraperitoneal laparoscopic radical prostatectomy. Eur Urol 65:610-619. https://doi.org/10.1016/j.eururo.2012.11.049

24. Porpiglia F, Fiori C, Bertolo R et al (2018) Five-year Outcomes for a Prospective Randomised Controlled Trial Comparing Laparoscopic and Robot-assisted Radical Prostatectomy. Eur Urol Focus 4:80-86. https://doi.org/10.1016/j.euf.2016.11.007

25. Ng CK, Kauffman EC, Lee MM et al (2010) A Comparison of Postoperative Complications in Open versus Robotic Cystectomy. Eur Urol 57:274-282. https://doi.org/10.1016/j.eururo.2009.06.001

26. Bochner BH, Dalbagni G, Sjoberg DD et al (2015) Comparing open radical cystectomy and robotassisted laparoscopic radical cystectomy: A randomized clinical trial. Eur Urol 67:1042-1050. https://doi.org/10.1016/j.eururo.2014.11.043

27. Khan MS, Gan C, Ahmed K et al (2016) A single-centre early phase randomised controlled three-arm trial of open, Robotic, and Laparoscopic Radical Cystectomy (CORAL). Eur Urol 69:613-621. https://doi.org/10.1016/j.eururo.2015.07.038

28. Gastecka A, Hnatyszyn-Dzikowska A, Hejka P et al (2018) Cost comparison of laparoscopic versus robot-assisted radical cystectomy. Heal Policy Technol 7:420-426.

https://doi.org/10.1016/j.hlpt.2018.10.008

29. Zhang S, Lin T, Zhang Q et al (2020) Comparison of perioperative outcomes in robot-assisted radical cystectomy and laparoscopic radical cystectomy. Int J Med Robot Comput Assist Surg 16. https://doi.org/10.1002/rcs.2074 
30. Kukreja JB, Metcalfe MJ, Qiao W et al (2020) Cost-Effectiveness of Robot-assisted Radical Cystectomy Using a Propensity-matched Cohort. Eur Urol Focus 6:88-94. https://doi.org/10.1016/j.euf.2018.07.001

31. Nazemi T, Galich A, Sterrett S et al (2006) Radical nephrectomy performed by open, laparoscopy with or without hand-assistance or Robotic methods by the same surgeon produces comparable perioperative results. Int Braz J Urol 32:15-22. https://doi.org/10.1590/S1677-55382006000100003

32. Hemal AK, Kumar A (2009) A prospective comparison of laparoscopic and robotic radical nephrectomy for T1-2N0M0 renal cell carcinoma. World J Urol 27:89-94. https://doi.org/10.1007/s00345-008-03219

33. Jeong IG, Khandwala YS, Kim JH et al (2017) Association of robotic-assisted vs laparoscopic radical nephrectomy with perioperative outcomes and health care costs, 2003 to 2015. JAMA - J Am Med Assoc 318:1561-1568. https://doi.org/10.1001/jama.2017.14586

34. Gershman B, Bukavina L, Chen Z et al (2020) The Association of Robot-assisted Versus Pure Laparoscopic Radical Nephrectomy with Perioperative Outcomes and Hospital Costs. Eur Urol Focus 6:305-312. https://doi.org/10.1016/j.euf.2018.10.004

35. Deane LA, Lee HJ, Box GN et al (2008) Robotic versus standard laparoscopic partial/wedge nephrectomy: A comparison of intraoperative and perioperative results from a single institution. J Endourol 22:947-952. https://doi.org/10.1089/end.2007.0376

36. Kural AR, Atug F, Tufek I, Akpinar H (2009) Robot-assisted partial nephrectomy versus laparoscopic partial nephrectomy: Comparison of outcomes. J Endourol 23:1491-1497. https://doi.org/10.1089/end.2009.0377

37. Benway BM, Bhayani SB, Rogers CG et al (2009) Robot Assisted Partial Nephrectomy Versus Laparoscopic Partial Nephrectomy for Renal Tumors: A Multi-Institutional Analysis of Perioperative Outcomes. J Urol 182:866-873. https://doi.org/10.1016/j.juro.2009.05.037

38. Wang AJ, Bhayani SB (2009) Robotic Partial Nephrectomy Versus Laparoscopic Partial Nephrectomy for Renal Cell Carcinoma: Single-Surgeon Analysis of > 100 Consecutive Procedures. Urology 73:306310. https://doi.org/10.1016/j.urology.2008.09.049

39. Lee S, Oh J, Hong SK et al (2011) Open versus robot-assisted partial nephrectomy: Effect on clinical outcome. In:Journal of Endourology. J Endourol, pp1181-1185

40. Pierorazio PM, Patel HD, Feng T et al (2011) Robotic-assisted versus traditional laparoscopic partial nephrectomy: Comparison of outcomes and evaluation of learning curve. Urology 78:813-819. https://doi.org/10.1016/j.urology.2011.04.065

41. Khalifeh A, Autorino R, Hillyer SP et al (2013) Comparative outcomes and assessment of trifecta in 500 robotic and laparoscopic partial nephrectomy cases: A single surgeon experience. J Urol 189:12361242. https://doi.org/10.1016/j.juro.2012.10.021

42. Trudeau V, Gandaglia G, Shiffmann J et al (2014) Robot-assisted versus laparoscopic nephroureterectomy for uppertract urothelial cancer: A population-based assessment of costs and perioperative outcomes. J Can Urol Assoc 8:E695-E701. https://doi.org/10.5489/cuaj.2051

43. Lee H, Kim HJ, Lee SE et al (2019) Comparison of oncological and perioperative outcomes of open, laparoscopic, and robotic nephroureterectomy approaches in patients with non-metastatic upper-tract 
urothelial carcinoma. PLoS ONE 14. https://doi.org/10.1371/journal.pone.0210401

44. Smith RP, Oliver JL, Peters CA (2011) Pediatric robotic extravesical ureteral reimplantation: Comparison with open surgery. J Urol 185:1876-1881. https://doi.org/10.1016/j.juro.2010.12.072

45. Schomburg JL, Haberman K, Willihnganz-Lawson KH, Shukla AR (2014) Robot-assisted laparoscopic ureteral reimplantation: A single surgeon comparison to open surgery. J Pediatr Urol 10:875-879. https://doi.org/10.1016/j.jpurol.2014.02.013

46. Kurtz MP, Leow JJ, Varda BK et al (2016) Robotic versus open pediatric ureteral reimplantation: Costs and complications from a nationwide sample. J Pediatr Urol 12. https://doi.org/10.1016/j.jpurol.2016.06.016. :408.e1-408.e6

47. Elizondo RA, Au JK, Song SH et al (2020) Open versus robot-assisted laparoscopic ureteral reimplantation: Hospital charges analysis and outcomes at a single institution. J Pediatr Surg. https://doi.org/10.1016/j.jpedsurg.2019.12.016

48. Brandao LF, Autorino R, Zargar H et al (2014) Robot-assisted laparoscopic adrenalectomy: Step-by-step technique and comparative outcomes. Eur Urol 66:898-905.

https://doi.org/10.1016/j.eururo.2014.04.003

49. Arghami A, Dy BM, Bingener J et al (2015) Single-port robotic-assisted adrenalectomy: Feasibility, safety, and cost-effectiveness. J Soc Laparoendosc Surg 19:1-5. https://doi.org/10.4293/JSLS.2014.00218

50. Probst KA, Ohlmann CH, Saar M et al (2016) Robot-assisted vs open adrenalectomy: evaluation of costeffectiveness and peri-operative outcome. BJU Int 118:952-957. https://doi.org/10.1111/bju.13529

51. Singh A, Jaipuria J, Goel A et al (2018) Comparing Outcomes of Robotic and Open Inguinal Lymph Node Dissection in Patients with Carcinoma of the Penis. J Urol 199:1518-1525. https://doi.org/10.1016/j.juro.2017.12.061

52. Yu H, Lu Y, Xiao Y et al (2019) Robot-assisted laparoscopic antegrade versus open inguinal lymphadenectomy: A retrospective controlled study. BMC Urol 19. https://doi.org/10.1186/s12894-0190571-4

53. McGuinness LA, Higgins JPT (2021) Risk-of-bias VISualization (robvis): An R package and Shiny web app for visualizing risk-of-bias assessments. Res Synth Methods 12:55-61. https://doi.org/10.1002/JRSM.1411

54. Tang K, Jiang K, Chen $\mathrm{H}$ et al (2017) Robotic vs. Retropubic radical prostatectomy in prostate cancer: A systematic review and an meta-analysis update. Oncotarget 8:32237-32257.

https://doi.org/10.18632/oncotarget.13332

55. Cao L, Yang Z, Qi L, Chen M (2019) Robot-assisted and laparoscopic vs open radical prostatectomy in clinically localized prostate cancer: perioperative, functional, and oncological outcomes: A Systematic review and meta-analysis. Med (Baltim) 98. https://doi.org/10.1097/MD.0000000000015770

56. Rai BP, Bondad J, Vasdev N et al (2020) Robot-assisted vs open radical cystectomy for bladder cancer in adults. BJU Int 125:765-779. https://doi.org/10.1111/BJU.14870

57. Kimura S, Iwata T, Foerster B et al (2019) Comparison of perioperative complications and health-related quality of life between robot-assisted and open radical cystectomy: A systematic review and meta- 
analysis. Int J Urol 26:760-774. https://doi.org/10.1111/IJU.14005

58. Iwata T, Kimura S, Foerster B et al (2019) Oncologic outcomes after robot-assisted versus open radical cystectomy: a systematic review and meta-analysis. World J Urol 37:1557-1570. https://doi.org/10.1007/s00345-019-02708-8

59. Crocerossa F, Carbonara U, Cantiello F et al (2021) Robot-assisted Radical Nephrectomy: A Systematic Review and Meta-analysis of Comparative Studies. Eur Urol 80:428-439

60. Li J, Peng L, Cao D et al (2020) Comparison of Perioperative Outcomes of Robot-Assisted vs. Laparoscopic Radical Nephrectomy: A Systematic Review and Meta-Analysis. Front Oncol 10:1789. https://doi.org/10.3389/FONC.2020.551052/BIBTEX

61. Xia L, Wang X, Xu T, Guzzo TJ (2017) Systematic Review and Meta-Analysis of Comparative Studies Reporting Perioperative Outcomes of Robot-Assisted Partial Nephrectomy Versus Open Partial Nephrectomy. J Endourol 31:893-909. https://doi.org/10.1089/END.2016.0351

62. Tsai SH, Tseng PT, Sherer BA et al (2019) Open versus robotic partial nephrectomy: Systematic review and meta-analysis of contemporary studies. Int J Med Robot Comput Assist Surg 15:e1963. https://doi.org/10.1002/RCS.1963

63. Chandrasekharam VVS, Babu R (2020) Robot-assisted laparoscopic extravesical versus conventional laparoscopic extravesical ureteric reimplantation for pediatric primary vesicoureteric reflux: a systematic review and meta-analysis. Pediatr Surg Int 36:1371-1378. https://doi.org/10.1007/S00383020-04749-2

64. Veccia A, Antonelli A, Francavilla S et al (2020) Robotic versus other nephroureterectomy techniques: a systematic review and meta-analysis of over 87,000 cases. World J Urol 38:845-852. https://doi.org/10.1007/S00345-019-03020-1

65. Tang K, Li H, Xia D et al (2015) Robot-assisted versus laparoscopic adrenalectomy: a systematic review and meta-analysis. J Laparoendosc Adv Surg Tech A 25:187-195. https://doi.org/10.1089/LAP.2014.0431

66. Ruurda JP, Visser PL, Broeders IAMJ (2003) Analysis of procedure time in robot-assisted surgery: Comparative study in laparoscopic cholecystectomy. Comput Aided Surg 8:24-29. https://doi.org/10.3109/10929080309146099

\section{Table 3}

Table 3 is available in the Supplemental Files section

\section{Figures}




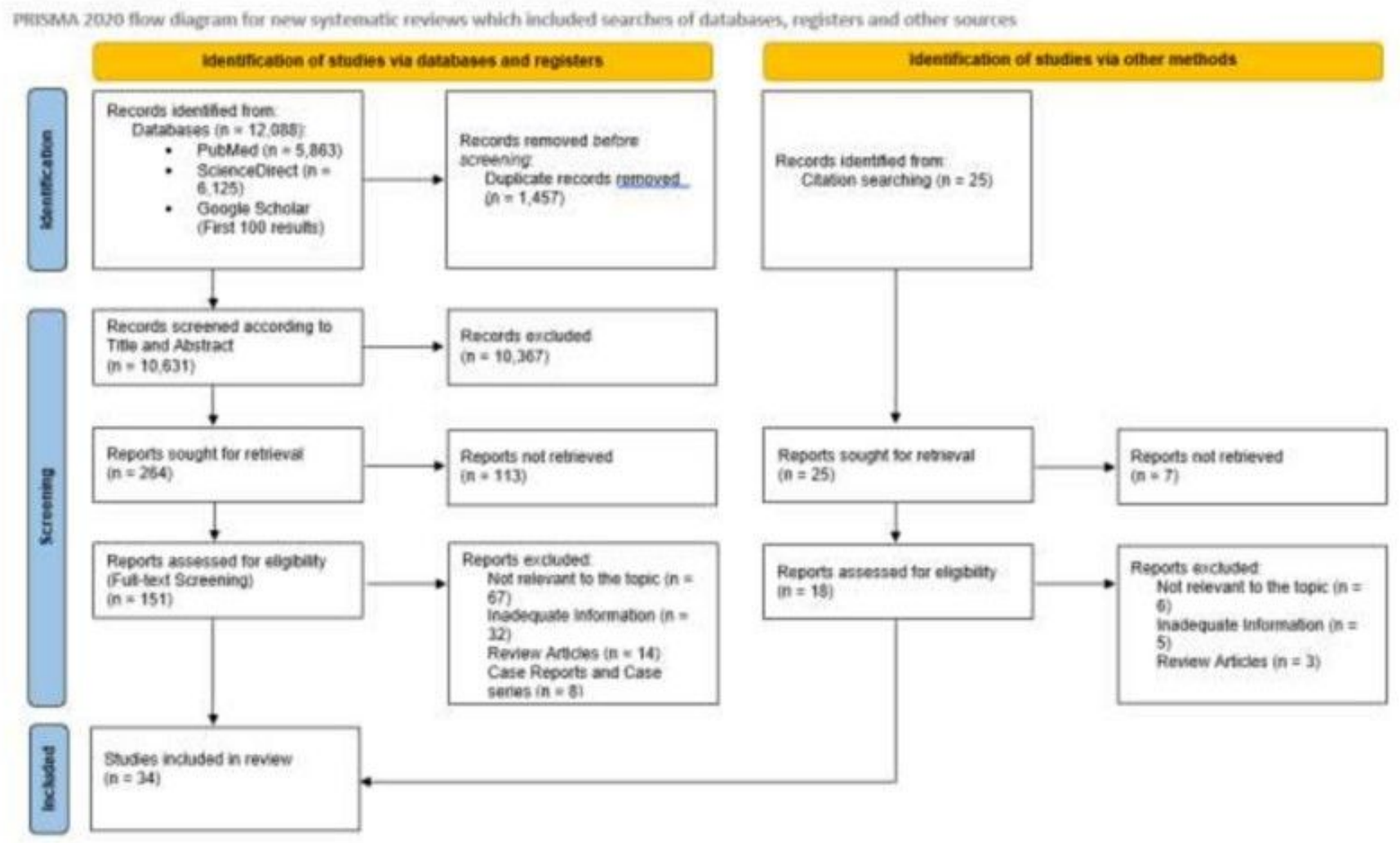

Figure 1: PRISMA flow diagram of included studies

Figure 1

See image above for figure legend. 




Figure 2: Risk of Bias (RoB) plot of RCTs

\section{Figure 2}

See image above for figure legend.

\section{Supplementary Files}

This is a list of supplementary files associated with this preprint. Click to download.

- Table3.docx 